\title{
Distributed Hybrid Spectrum Access for Cognitive Femtocells in 5G Mobile Networks
}

\author{
Pavel Mach $^{1}$, Zdenek Becvar ${ }^{1}$, Michal Vondra ${ }^{1}$ \\ ${ }^{1}$ Czech Technical University in Prague, Czech Republic, \\ Technicka 2, 16627 Prague, Czech Republic \\ machp2@fel.cvut.cz
}

\begin{abstract}
The femto access points (FAPs) with cognitive capabilities, also known as cognitive FAPs, are able to efficiently mitigate interference in two-tier heterogeneous networks. Hence, the concept of cognitive FAPs can be seen as one of the key enablers for future 5G networks, where high density of FAPs is foreseen. However, conventional overlay and underlay spectrum sharing strategies enabling the cognitive FAPs to access spectrum of macrocells have several drawbacks. The main disadvantage of the former one is that its efficiency fully depends on the activity of macrocell users and insufficient resources can remain for the users of the FAP. The main weakness of the latter one is that it can result in low transmission efficiency because transmission power level of the FAPs is restricted. In this paper, we propose a novel hybrid spectrum sharing that allows the FAPs to use both overlay and underlay strategies simultaneously and, thus, increase performance of FAPs' users. The proposed scheme is fully distributed since the FAPs allocate resources autonomously. The results show that the proposed algorithm is able to significantly outperform competitive schemes in terms of served traffic for femtocell users and, simultaneously, served traffic for macrocell users is intact.
\end{abstract}

Index Terms-Cognitive femtocells; $5 \mathrm{G}$; overlay/underlay spectrum sharing.

\section{INTRODUCTION}

According to [1], incremental enhancement of 4G-based mobile networks will not be able to satisfy increasing demands and requirements of users on wireless mobile networks. Hence, 5G mobile networks will emerge in near future. Key technological concepts enabling huge data rate transmissions by means of $5 \mathrm{G}$ networks are [2]: i) extreme densification of base stations managed by small cells deployment, ii) increased bandwidth by utilization of millimetre waves, and iii) increased spectral efficiency through massive MIMO techniques [3].

To fully exploit millimetre waves and massive MIMO for the purpose of $5 \mathrm{G}$ while keep the equipment cost minimal, many challenges need to be still overcome. On the other hand, dense deployment of small cells, mainly represented by femto access points (FAPs), is relatively cheap and matured approach to increase capacity of contemporary wireless networks. However, densification of the FAPs

Manuscript received 23 October, 2015; accepted 14 December, 2015.

This work has been supported by Grant No. 13-24931P funded by the Czech Science Foundation. inevitably results in severe interference to the overlay macrocells. Moreover, the mutual interference among the FAPs cannot be ignored as well. The interference problem is critical especially if the FAPs use closed access regime [4] and if co-channel deployment is considered [5]. A promising option for interference avoidance is to enhance the FAPs with cognitive capabilities towards "cognitive FAPs" [6]. In a system with cognitive FAPs, the macrocell base stations (MBSs) are assumed to be the primary users (PUs) while the cognitive FAPs are considered to be the secondary users (SUs). Thus, the MBSs have higher priority in usage of radio resources than the FAPs and the MBS should not be interfered by the FAP's transmission. The cognitive FAPs may access the spectrum assigned primarily to the MBS in an underlay or an overlay approach [7], [8] (note that the overlay spectrum sharing used by cognitive FAPs is sometimes referred to as an interweave approach [9]).

If the underlay spectrum sharing (USS) is exploited, the FAPs may use the same spectrum with the MBS as long as the interference is kept under a predefined threshold. Basically, the schemes utilizing various power control techniques at the side of the FAPs in order to mitigate interference to the MBS can be considered as a specific case of the USS. This option has been recently thoroughly investigated in current research literature (see, e.g., [10][12]). The power control techniques utilizing the cognitive principle (especially the sensing techniques) adopting the USS have been introduced, for example, in [13], [14]. The authors in [13] propose downlink transmit power allocation on each channel exploiting information on downlink radio resource usage obtained from sensing. Similarly, the optimal power allocation in order to minimize interference to the PUs is proposed in [14]. In general, the main drawback of the USS is that the femtocell users (FUEs) may not be able to attach to the FAP as its coverage is limited because of restricted transmission power of the FAP. Moreover, reduced transmission power also results in utilization of less effective modulation and coding scheme (MCS), which has negative impact on spectral efficiency of the whole system.

On the other hand if the overlay spectrum sharing (OSS) is exploited, the FAPs access only radio resources that are not currently utilized by the MBS [15]-[19]. In [15], the FAPs autonomously sense the radio frequencies used by the MBS and schedule their transmission to unoccupied radio resources. A dynamic spectrum reuse is proposed in [16]. If 
the macrocell user (MUE) is close to the FAP and suffers from FAP's interference, the FAP decides whether to occupy these frequencies or not. In [17], the Gale-Shapley Spectrum Sharing (GSOIA) scheme is suggested. The GSOIA is based on multichannel opportunistic sensing resulting in no collisions among the MBS and the FAPs. The authors in [18] proposes Cognitive Hybrid Division Duplex (CHDD) where the MBSs use FDD (Frequency Division Duplex) while the FAPs access the available band by means of TDD (Time Division Duplex). Another OSS scheme is presented in [19], where the FAPs perform sensing process and then access only those channels that are assumed to be unoccupied (i.e., not utilized by the MBS and its users). These channels are then accessed opportunistically based on traffic pattern from the MBS. A specific case of the OSS is considered in [20], where the MBSs and the FAPs use mutually exclusive sub-channels by means of fractional spectrum reuse. This approach, however, decreases the spectral efficiency. Generally, the main disadvantage of the OSS proposed for cognitive FAPs is that the FAPs are strongly dependent on the activity of the MBSs. In the worst case scenario, there could be none or only limited amount of resources available to the FAPs if the MBS is loaded heavily.

Two recent studies in the literature proposed a hybrid spectrum sharing (HSS) for cognitive FAPs combining both above-mentioned OSS and USS [21], [22]. In [21], we have proposed fully distributed scheme that change dynamically between the OSS and the USS if beneficial to the FAP' users (FUE) in terms of throughput. In [22], the authors propose a centralized scheme allowing to use both the USS and the OSS simultaneously. The FAPs use primarily resources in the OSS and if these are not sufficient, resources in the USS (occupied by the MBS) are exploited. The disadvantage of [22] is its high complexity and that it could generate significant amount of overhead due to centralized approach. With respect to [22] we propose fully distributed HSS scheme distinguished by low complexity and low signalling overhead. Moreover, we propose that the FAPs primarily utilize the USS since all FAPs can use them while the resources in the OSS have to be shared by neighbouring FAPs to avoid interference. Hence, some FAPs may manage only with resources in the USS and more resources in the OSS can be used by highly loaded FAPs. As demonstrated by the results, this approach yields higher throughput for the FUEs when compared to [22].

The rest of this paper is organized as follows. The next section introduces a system model used for the proposal description. Section III is focused on the proposed hybrid spectrum sharing scheme for the cognitive FAPs. The simulation methodology and simulation results are described in Section IV and Section V, respectively. The last section concludes the paper.

\section{SySTEM MODEL}

We define system model with seven MBSs in a hexagonal grid (note that the example of system model depicted in Fig. 1 shows only central MBS for clarity purposes). Within coverage of each MBS, $Y$ FAPs are deployed. Both the
MBSs and the FAPs adopt OFDMA-based system where available radio resources are divided into resource blocks (RBs). In OFDMA systems based on LTE-A the amount of all RBs available within a frame at physical layer, $n_{R B}$, is between 120 and 2200 depending on bandwidth.

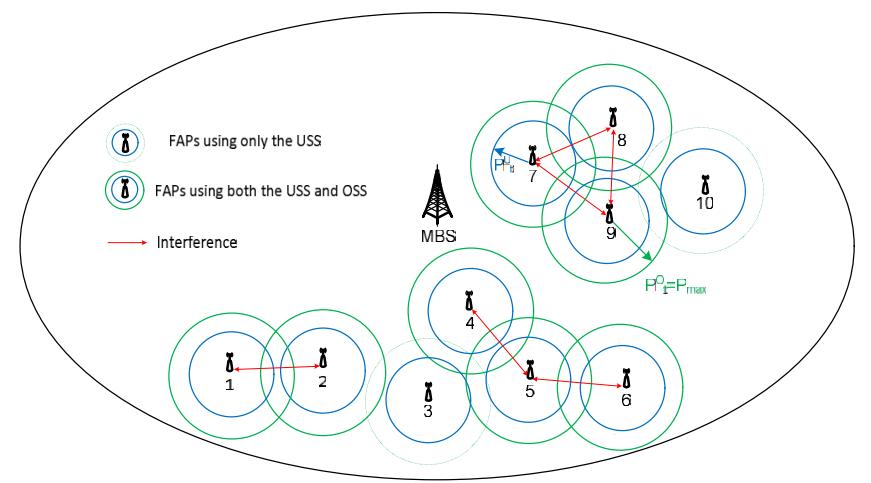

Fig. 1. Example of system model.

In proposed HSS, some of the available RBs can be dedicated to the FUEs in the USS while others RBs may be simultaneously exploited in the OSS. The transmission power used at the RBs in the USS and the OSS differs. In the USS, we set transmission power of $i$-th FAP $\left(p_{, t . i, U}\right)$, to guarantee that SINR of the macrocell users (MUEs) $\left(\gamma_{m}\right)$ is degraded, in the worst case scenario, only by $\delta$. Hence, $p_{\text {,t.i, } U}$ is set as

$$
p_{t, i, U}->\gamma_{m} \geq \gamma_{m(\mathrm{noFAP})}-\delta
$$

where $\gamma_{m \text { (noFAP) }}$ is SINR of the MUEs if no FAPs are introduced, and $\delta$ stands for allowable decrease in $\gamma_{m}$. We consider that $\delta=3 \mathrm{~dB}$, but its value could be set depending on the operator's preferences (e.g., $\delta=0 \mathrm{~dB}$ allows no decrease of MUEs performance at all). In case of the OSS, the interference to the MUEs is avoided by allocation of orthogonal RBs to the FAPs. Hence, the transmission power by the $i$-th FAP in the OSS $\left(p_{\text {,t.i,O }}\right)$ is not restricted and its value is set always to maximum $\left(P_{\max }\right)$.

The SINR of the $f$-th FUE at the RBs used in the USS $\left(\gamma_{f i, U}\right)$ and the OSS $\left(\gamma_{f i, O}\right)$, and SINR of $m$-th MUE $\left(\gamma_{m}\right)$ is expressed as:

$$
\begin{gathered}
\gamma_{f i, U}=\frac{g_{f i} p_{t, i, U}}{N I+\sum_{\substack{y=1 \\
y \neq i}}^{Y} g_{f y} p_{t, i, U}+g_{f b} P_{t}}, \\
\gamma_{f i, O}=\frac{g_{f i} p_{t, i, O}}{N I+\sum_{\substack{y=1 \\
y \neq i}}^{Y} g_{f y} p_{t, i, O}}, \\
\gamma_{m}=\frac{g_{m b} P_{t}}{N I+\sum_{y=1}^{Y} g_{m y} p_{t, i, U}},
\end{gathered}
$$

where $P_{t}$ is the transmission power of the MBS, NI represents noise plus interference from the MBS, $g_{f i}, g_{f y}, g_{f b}$, $g_{m b}$, and $g_{m y}$ are the channel gains between the FUE and its serving FAP, the FUE and individual interfering FAPs in the USS, the FUE and the MBS, the MUE and the MBS, and the MUE and the FAP, respectively. In case of $\gamma_{f i, U}$, the 
interference to the $\underline{i}$-th FAP originates from all the FAPs using the USS since this mode is used every time when the FAP is active. In case of $\gamma_{f i, O}$, the interference is caused only by the FAPs, which are not direct neighbours and which are using the same RBs in the OSS. The reason is that the direct neighbours use orthogonal resources and do not interfere with FAPs in the OSS. The FAP is considered as the direct neighbour if it can cause harmful interference while transmitting with $P_{\max }$. Consequently, $i$-th FAP is the direct neighbour of $j$-th FAP if

$$
g_{i j} P_{\max } \geq N I-\sigma_{n}
$$

where $g_{i j}$ is the channel gain between the FAPs and $\sigma_{n}$ is the direct neighbourhood interference threshold. If the $\sigma_{n}$ is set to be low (or even negative), the FAPs have low number of direct neighbours but high amount of interference can be generated between them in the OSS. On the other hand, higher value of $\sigma_{n}$ protects the FAPs against interference in the OSS. A disadvantage of high $\sigma_{n}$ is that each FAP has usually more direct neighbours resulting in less amount of resources available in the OSS. In our model, direct neighbours are determined by means of graph theory. We denote each graph as $G_{x}=\left(V_{x}, V_{x}\right)$, where $V_{x}$ is the amount of FAPs in the $x$-th graph and $E_{x}$ represents the connection between FAPs corresponding to potential interference from the direct neighbours. The connection between direct neighbours is expressed by a direct neighbourhood matrix, which is shown in Fig. 2 (this matrix is derived from deployment shown in Fig. 1). The direct neighbours of the FAPs are represented by value " 1 " in the matrix if (5) is fulfilled. The direct neighbourhood matrix is changed only if new FAP is added, existing FAP is removed, or if the FAP is switched on/off ( $P_{\max }$ is assumed to be unchanged).

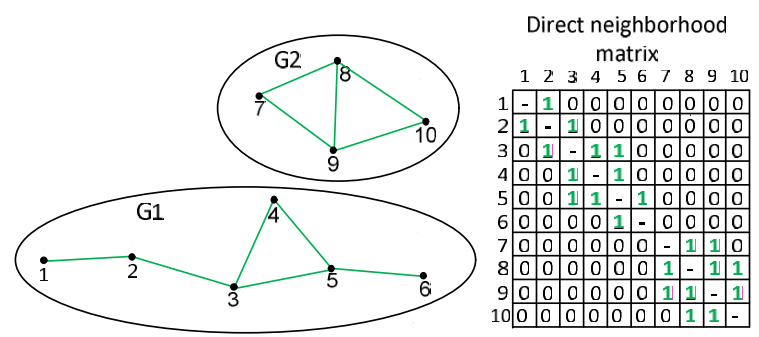

Fig. 2. Determination of direct neighbours and creation of direct neighbourhood matrix (location of FAPs and their neighbourhood is taken from Fig. 1).

To determine which RBs are occupied either by the MBS or the FAPs, each FAP is assumed to have sensing capability using the energy detection. This sensing method is the most common due to its low computational and implementation complexities [13]. The sensing is supposed to be performed periodically in frames dedicated for this purpose according to [8]. During the sensing frames, the FAP determines the received interference power at individual RBs. If the interference power at the $r$-th RB $\left(l_{r}\right)$ is above the sensing interference threshold $\sigma_{s}$, the RB is supposed to be occupied either by the MBS or the FAPs in the OSS.

\section{Proposed Hybrid SPeCtrum SHARING}

The basic principle of the proposed HSS is depicted in Fig. 3 where, without loss of generality, two direct neighbouring FAPs are assumed. In case of the USS, the FAPs can use all the RBs but its transmission power has to be restricted according to (1). Contrary, if the OSS is exploited, the FAP can access only RBs not currently utilized by the MBS in order to avoid interference since $P_{\max }$ is considered. Moreover, direct neighbouring FAPs have to use orthogonal RBs in the OSS. A distinguishable feature of the proposed HSS is that the FAP may use some RBs in the USS while other RBs are exploited in the OSS at the same time. As shown in Fig. 1, the RBs occupied by the MBS are used in the USS while the unoccupied RBs can be accessed in the OSS without restriction on transmission power. We propose to use the RBs in the USS by the FAPs preferentially (contrary to [22]). Only if RBs in the USS are not sufficient for the FAP, the FAP may use additional RBs in the OSS. The reason why RBs in the USS are used with priority is that all the FAPs can exploit them while the same RBs cannot be utilized by direct neighbours in the OSS due to interference (Fig. 3). We assume the FAPs in direct neighbourhood share by default the RBs in the OSS with equal priority so that the amount of RBs in the OSS for each FAP is

$$
n_{R B(a), O}=\frac{n_{R B}-n_{R B, m}}{1+n_{N i}},
$$

where $n_{R B, m}$ is the amount of RBs used by the MBS and $n_{N i}$ is the number of direct neighbours of the $i$-th FAP using some RBs in the OSS. If some FAPs use currently less RBs in the OSS than $n_{R B(a), O}$, the direct neighbouring FAPs with high requirements on RBs can borrow these (in Fig. 3, FAP2 borrows two RBs from FAP1). To summarize, the giving preferences to utilization of RBs in the USS increases $n_{R B(a), O}$ by lowering $n_{N i}$ since lightly loaded FAPs can handle users requirements only with the RBs in the USS (or less RBs in the OSS are needed). Consequently, more heavily loaded FAPs can share higher amount of RBs in the OSS and more traffic is served by them. Note that to obtain $n_{N i}$, the FAPs have to advertise its direct neighbours whenever it start/stop using the RBs in the OSS.

The aim of the proposed HSS is that the FAPs are able to allocate RBs in fully distributed manner. This is enabled by FAPs' sensing capabilities since sensing allows to find out, which RBs are used by the MBS and direct neighbouring FAPs in the OSS. As described in previous section, the sensing is done periodically in so-called sensing frames where no data is transmitted as all RBs in the sensing frame are utilized for the sensing. To determine RBs, which are utilized by the direct neighbouring FAPs in the OSS, we propose that the sensing frames of individual neighbouring FAPs should not overlap. To that end, the FAP newly deployed into the network negotiates sensing frames with its direct neighbours, which are found according to (5). 


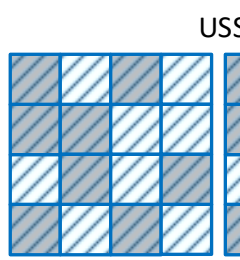

FAP 1
USS

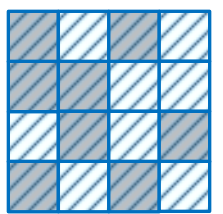

FAP 2
OSS

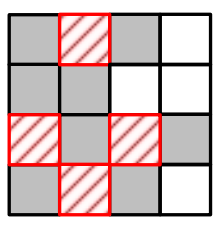

FAP 1

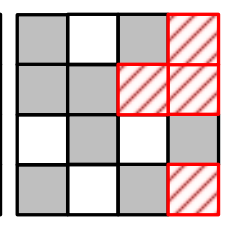

FAP 2

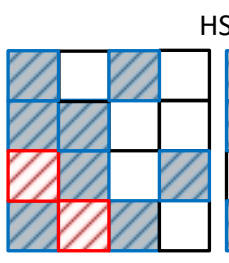

FAP 1
HSS

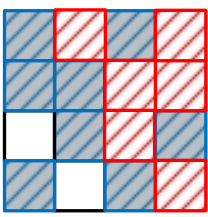

FAP 2

Fig. 3. The allocation of RBs in case of conventional USS and OSS in comparison to proposed HSS.

The allocation of the RBs by the FAP in the HSS is done by Algorithm 1. During the sensing, the FAP acquires knowledge on the amount of RBs available in the USS $\left(n_{R B(a), U}\right)$ and the amount of RBs in the OSS that are not occupied at the moment by its direct neighbours $\left(n_{R B(n o), O}\right)$. Then, the FAP calculates downlink requirements of all its active FUEs on RBs in the USS per frame as (in Algorithm 1, line 2)

$$
n_{R B(r), U}=\sum_{f=1}^{z} \operatorname{ceil}\left(\frac{\xi_{f i}}{\Lambda \Gamma_{f i, U}}\right),
$$

where $z$ is the amount of active FUEs, $\xi_{f i}$ is the amount of data (in bits) sent in downlink to the $f$-th FUE per frame, $\Lambda$ is the amount of resource elements (REs) per RB, and $\Gamma_{f, U}$ corresponds to the transmission efficiency of the $f$-th FUE using the RBs in the USS. The transmission efficiency is measured by the amount of transmitted bits per RE depending on selected MCS, which is assigned according to SINR [23], [24].

The FAP serves preferably all its FUEs in the USS (see lines 3-4). If not enough resources are available in the USS, the FAP has to utilize all RBs available in the USS together with some RBs in the OSS. Then, the FAP calculates the number of RBs needed in the OSS after all RBs in the USS are allocated as

$$
n_{R B(r), O}=\sum_{f=1}^{z_{O}} \operatorname{ceil}\left(\frac{\xi_{f i}-\xi_{f i, U}}{\Lambda \Gamma_{f i, O}}\right),
$$

where $z_{O}$ represents the number of active FUEs that still need to be served in the OSS after allocation of RBs in the USS, the term $\xi_{f, U}$ corresponds to the amount of data that is served in the USS, and $\Gamma_{f i, O}$ corresponds to the transmission efficiency of the $f$-th FUE using the RBs in the OSS. If the number of required RBs in the OSS is lower than the number of RBs not currently occupied by its direct neighbours, the FAP assigns necessary amount of RBs in the OSS to the FUEs (lines 8-9). Otherwise, if the FAP needs more RBs than available at the moment while more RBs in the OSS should be available to it (i.e., if $n_{R B(r), O} \leq n_{R B(a), O}$ ), the FAP sends a message to all its direct neighbours requesting to release specific amount of RBs (line 11). After these RBs are released, the FAP exploits them in the OSS (line 12). Note that the message itself contains information on how many RBs should be released and when.

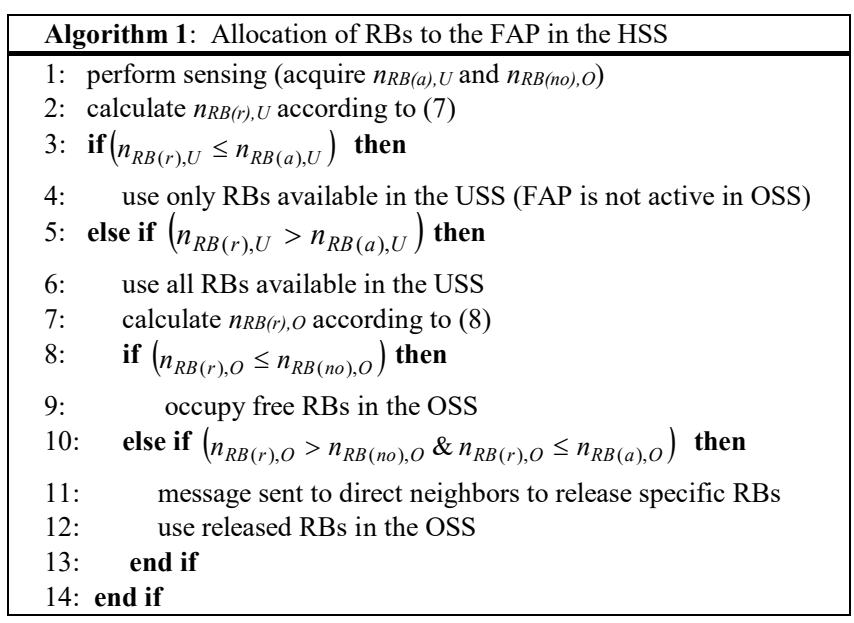

\section{Simulation SCENARIOS AND MODElS}

The simulations are performed in MATLAB. We have used own-developed simulator based on FDD LTE-A release 12 with parameters' set-up aligned with Small cell forum as presented in Table I. We model with 7 MBSs in hexagonal grid, dual strip model [25] with 20 FAPs randomly placed in 40 apartments (see example in Fig. 4, where six neighbouring MBSs are not depicted for the sake of simplicity). The simulations are done for 10 drops (the FAPs' locations are generated randomly for each drop). Each drop lasts for 10000 s real time and final results are averaged out over time and drops. Note that 10 drops have been sufficient in our simulation model since the result for 5 and more drops are nearly the same. In addition, the simulations are averaged out for two locations of dual strip block, i) near the MBS where RSS (received signal strength) from adjacent MBSs is $-90 \mathrm{dBm}$ at average and ii) at the edge of the MBS cell where RSS from adjacent MBSs is $-70 \mathrm{dBm}$ at average.

We assume 20 MUEs moving along the sidewalk (see Fig. 4). Initial position and movement direction of each MUE is selected randomly. After that, the MUEs are moving along straight trajectories with speed of $1 \mathrm{~m} / \mathrm{s}$. If the MUE leaves the MUE's restricted area, a new MUE enters the scenario at the opposite side. The FUEs are moving according to the mobility model specified in [26], where the movement is limited by apartment boundaries. The indoor path loss is calculated according to ITU-R P.1238 model. For outdoor path loss, COST 231 empirical model is used [27].

In the simulation, we consider realistic FTP model for the FUEs. During first simulation cycle (10 000s), the FUEs 
generate traffic load resulting in mean load of the FAP load equal to $1 \mathrm{Mbit} / \mathrm{s}$ with maximal load of $2.5 \mathrm{Mbit} / \mathrm{s}$. After that traffic load is gradually increased for each simulation cycle up to $15 \mathrm{Mbit} / \mathrm{s}$ mean load of the FAP and maximal load of 37.5 Mbit/s.

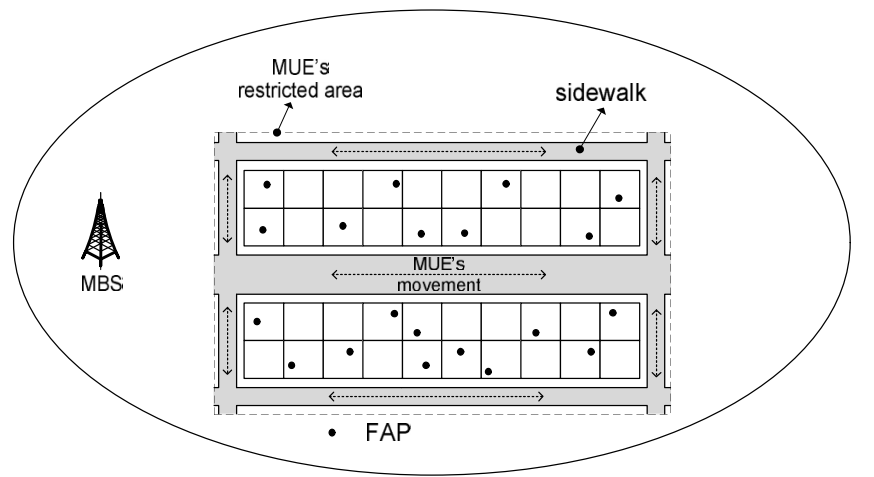

Fig. 4. Simulation scenario.

TABLE I. SUMMARIZATION OF SIMULATION PARAMETERS

\begin{tabular}{|c|c|}
\hline Parameter & VALUE \\
\hline Carrier frequency $f[\mathrm{GHz}]$ & 2.0 \\
\hline MBS/FAP channel bandwidth $B W[\mathrm{MHz}]$ & $20 / 20$ \\
\hline Max./Min. FAP transmit power $P_{\max }[\mathrm{dBm}]$ & $10 /-20$ \\
\hline MBS transmit power $[\mathrm{dBm}]$ & 43 \\
\hline Noise [W] & $\mathrm{BW} \cdot 4 \cdot \mathrm{pW} / \mathrm{GHz}$ \\
\hline Number of FAPs/FUEs/MUEs [-] & $20 / 40 / 20$ \\
\hline Loss of internal wall, external wall, window [dB] & $5,10,3$ \\
\hline$S I N R_{\min }, \Delta_{f}, \sigma_{n}[\mathrm{~dB}]$ & $-9.478[24], 4,10$ \\
\hline $\begin{array}{l}\text { Mean/Max load of FAP (generated by FTP } \\
\text { model) }[\mathrm{Mbit} / \mathrm{s}]\end{array}$ & $1-15 / 2.5-37.5$ \\
\hline Sensing period $[\mathrm{s}]$ & 0.2 \\
\hline Indoor path loss model & ITU-RP.1238 [27] \\
\hline Outdoor path loss model & COST $231[27]$ \\
\hline Simulation time $[\mathrm{s}] /$ Number of drops [-] & $10000 / 10$ \\
\hline
\end{tabular}

The activity of the MUEs is modelled similarly as in case of the FUEs by means of FTP traffic model. For the purpose of our simulation, the mean load of the MBS varies from $0 \%$ to $100 \%$. While the former case represents one extreme scenario when no MUEs activity occurs, the latter corresponds to the opposite extreme scenario when the MBS is fully loaded all the time and the FAPs have to access all RBs in the conventional USS mode.

\section{Simulation Results}

The performance of proposed HSS (HSS-prop.) is compared to the conventional USS based on [13], [14], the OSS base on [15]-[19], the HSS proposed in [21] (denoted as HSS-EW), and the proposed in [22] (HSS-TWC).

Figure 5 shows the amount of data served for both the FUEs and the MUEs if FAPs load is varying. The observed gain of the HSS-prop. in terms of FUE's performance with respect to other competitive schemes is up to $38.8 \%$ (compared to the OSS), $29.1 \%$ (compared to the USS), $11.8 \%$ (compared to the HSS-EW), and $7.9 \%$ (compared to the HSS-TWC). Moreover, it is demonstrated that the MUEs performance is not degraded by any scheme since the MBS is not fully loaded and can still serve all requests of the MUEs.
Figure 6 illustrates the performance of individual schemes for different mean MBS load while the FAPs' load remains constant. If the load of the MBS is $0 \%$, the HSS-prop. outperforms the USS by $29.8 \%$ and the HSS-EW by $3.3 \%$ while the HSS-TWC and the OSS perform the same in terms of FUEs' served data. In the opposite extreme all schemes perform the same $(63.4 \%$ is of FUEs' data is served) but the OSS ( $0 \%$ of data is served). If load of the MBS is between extreme cases, proposed HSS always outperforms all other conventional competitive schemes by up to $30.8 \%$ (USS), $60.4 \%$ (OSS), $11.3 \%$ (HSS-EW), and $7.3 \%$ (HSS-TWC). Regarding the performance of the MUEs, the MBS serves all MUEs traffic for all algorithms as long as the load of the MBS is below $80 \%$. For higher MBS's load, the MUEs QoS is degraded by up $2.6 \%$ for all considered schemes but the OSS, which does not impair the performance of the MUEs.

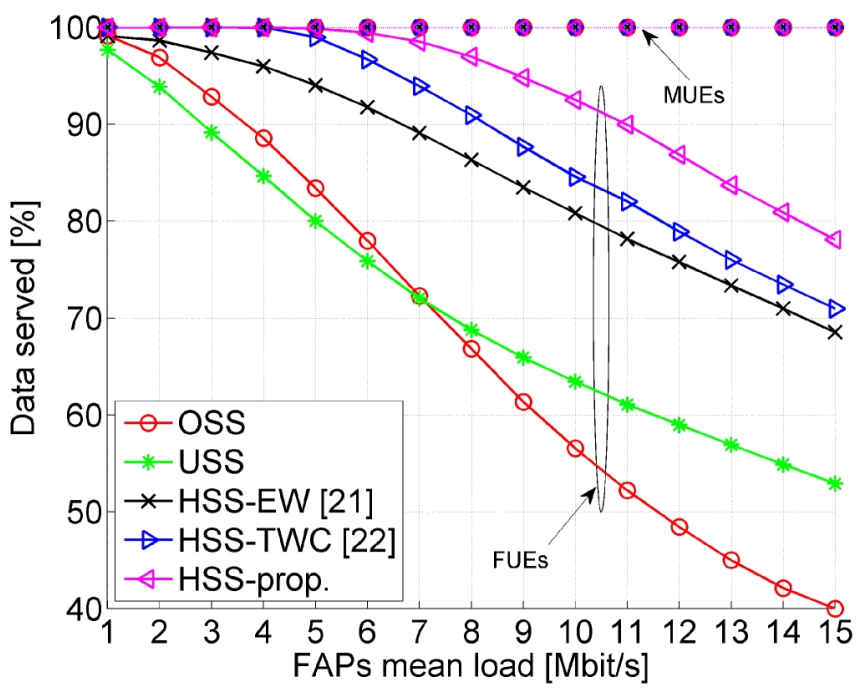

Fig. 5. The amount of data served (both the FUEs and the MUEs) depending on FAPs load while MBS load is at $50 \%$.

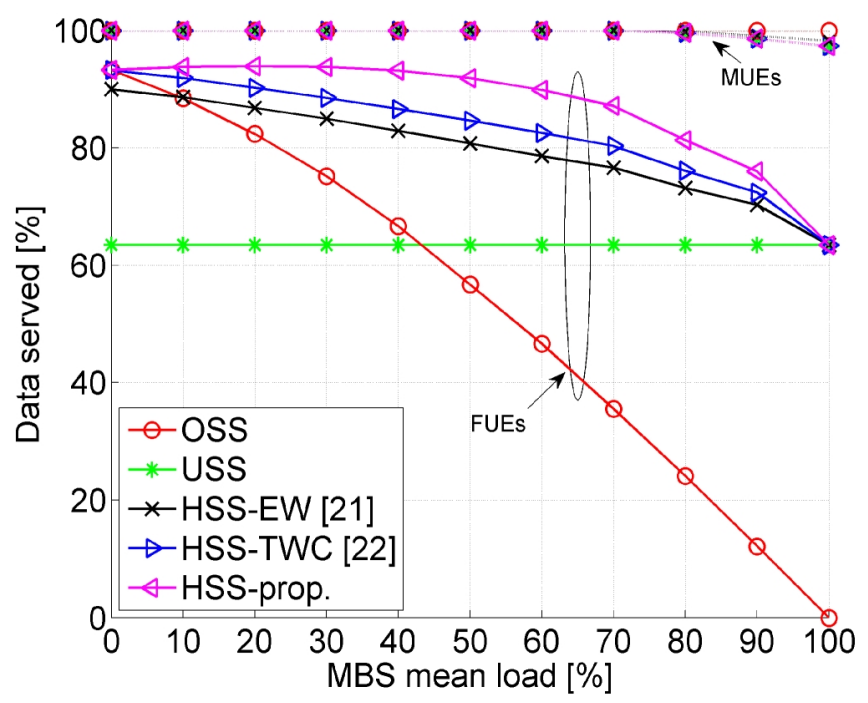

Fig. 6. The amount of data served (both the FUEs and the MUEs) depending on MBS load while FAPs load is fixed to $10 \mathrm{Mbit} / \mathrm{s}$.

Figure 7 and Fig. 8 show additional overhead generated by all HSS schemes over the backhaul when compared to the OSS and the USS. Note the size of each signalling message with consideration of overhead from all layers of protocol stack is approximately 800 bits [28]. 


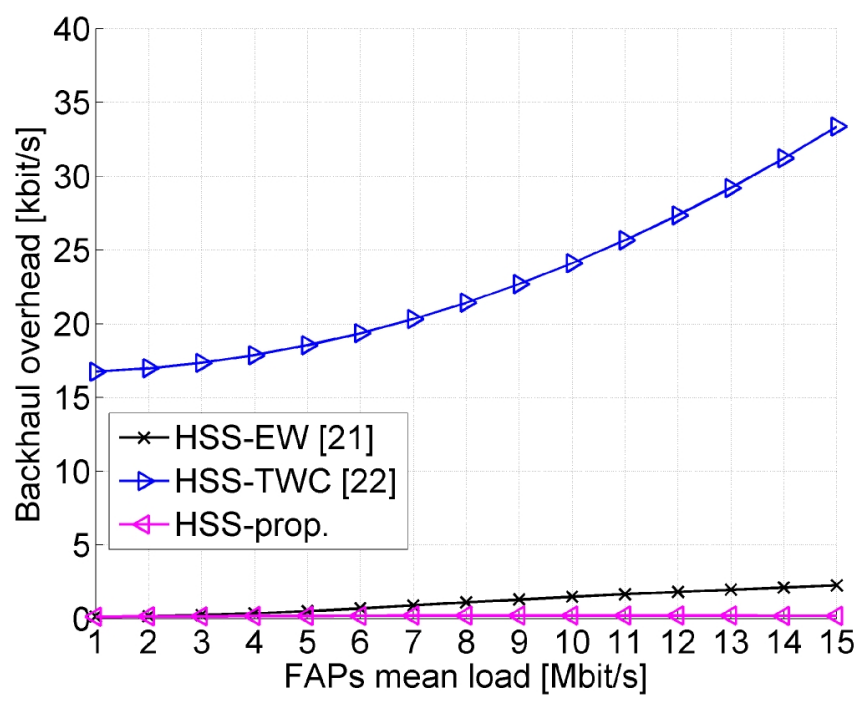

Fig. 7. The amount of backhaul overhead depending on FAPs load while MBS load is at $50 \%$.

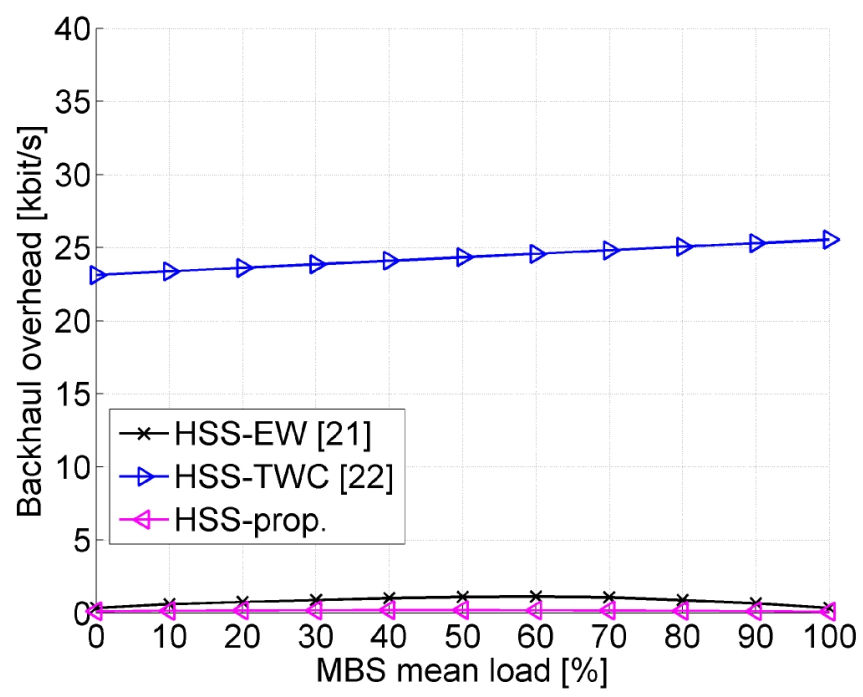

Fig. 8. The amount of backhaul overhead depending on MBS load while FAPs load is fixed to $10 \mathrm{Mbit} / \mathrm{s}$.

The highest backhaul overhead is generated be centralized HSS-TWC scheme and is up to $33.3 \mathrm{kbit} / \mathrm{s}$. Contrary, the distributed schemes are distinguished by significantly lower backhaul overhead when compared to the HSS-TWC. To be more precise, the HSS-EW generates up to $2.25 \mathrm{kbit} / \mathrm{s}$ and the HSS-prop. only up to $0.2 \mathrm{kbit} / \mathrm{s}$.

\section{CONCLUSIONS}

In this paper, we have proposed novel hybrid spectrum sharing scheme for cognitive femtocells, where the femtocells are able to utilize radio resources in both the underlay and overlay sharing approach at the same time. In this regard we have proposed a fully distributed hybrid spectrum sharing scheme, which efficiently allocates radio resources to individual FAPs. The results show that the proposed scheme significantly outperforms other competitive schemes in terms of served traffic for the FUEs while the interference to the MUEs is sufficiently mitigated so the served traffic for the MUEs is not impaired. Further, it is shown that the signalling overhead is kept at minimum. Especially, if we compare our proposal to similar scheme presented in [22], we are able to outperform this scheme in terms of FUEs throughput due to the fact that the FAPs primarily occupy resources in the USS. Hence, more resources are then available for heavily loaded FAPs in the OSS.

The proposed scheme can be enhanced in the future by smart allocation of RBs to the FUEs when FUEs with weak channel quality should utilize the OSS while FUEs close to the FAP can use the USS.

\section{REFERENCES}

[1] Cisco, Visual Networking Index, white paper at Cisco.com, 2014

[2] J. G. Andrews, et. al, "What will 5G be?", IEEE J. Sel. Areas Commun., vol. 32, no. 6, pp. 1065-1082, 2014. [Online]. Available: https://doi.org/10.1109/JSAC.2014.2328098

[3] A. L. Swindlehurst, E. Ayanoglu, P. Heydari, F. Capolino, "Millimeter-wave massive MIMO: the next wireless revolution?", IEEE Commun. Mag, vol. 52, no. 9, pp. 56-62, 2014. [Online] Available: https://doi.org/10.1109/MCOM.2014.6894453

[4] A. Golaup, M. Mustapha, L. B. Patanapongpibul, "Femtocell access control strategy in UMTS and LTE", IEEE Commun. Mag, vol. 47, no. 9, pp. 117-123, 2009. [Online]. Available: https://doi.org/ 10.1109/MCOM.2009.5277464

[5] V. Chandrasekhar, J. G, Andrews, "Spectrum allocation in shared cellular network", IEEE Trans. Commun., vol. 57, no 10, pp. 30593068, 2009. [Online]. Available: https://doi.org/10.1109/ TCOMM.2009.10.080529

[6] S. A. Rubaye, A. A. Dulaimi, J. Cosmas, "Cognitive femtocells", IEEE Vehicular Technology Magazine, vol. 6, no. 1, pp. 44-51, 2011. [Online]. Available: https://doi.org/10.1109/MVT.2010. 939902

[7] W. Wang, G. Yu, A. Huang, "Cognitive radio enhanced interference coordination for femtocell networks", IEEE Communications Magazine, vol. 51, no. 6, pp. 37-43, 2013. [Online]. Available: https://doi.org/10.1109/MCOM.2013.6525593

[8] A. U. Ahmed, M. T. Islam, M. Ismail, "A review on femtocell and its diverse mitigation techniques in heterogeneous network", Wireless Personal Communications, vol. 78, pp. 85-106, 2014. [Online] Available: https://doi.org/10.1007/s11277-014-1737-8

[9] H. O. Kpojime, G. A. Safdar, "Interference mitigation in cognitive radio based femtocells", accepted in IEEE Communications Surveys \& Tutorials, 2014

[10] H. Saad, A. Mohamed, T. ElBatt, "A cooperative Q-learning approach for online power allocation in femtocell networks", IEEE Vehicular Technology Conf. (VTC fall), 2013, pp. 1-6. [Online] Available: https://doi.org/10.1109/vtcfall.2013.6692027

[11] H. Wang, R. Song, "Distributed Q-learning for interference mitigation in self-organised femtocell networks: synchronous or asynchronous?", Wireless Personal Communications, vol. 71, no. 4, pp. 2491-2506, 2013. [Online]. Available: https://doi.org/10.1007/ s11277-012-0950-6

[12] R. Kurda, L. Boukhatem, T. A. Yahiya, M. Kaneko, "Power adjustment mechanism using context information for interference mitigation in two-tier heterogeneous networks", IEEE Symposium on Computers and Communications (ISCC), 2014, pp. 1-6. [Online]. Available: https://doi.org/10.1109/iscc.2014.6912532

[13] D. Sun, X. Zhu, Z. Zeng, S. Wan, "Downlink power control in cognitive femtocell networks", Int. Conf. Wireless Communications and Signal Processing (WCSP), 2011, pp. 1-5. [Online]. Available: https://doi.org/10.1109/wcsp.2011.6096947

[14] X. Tao, Z. Zhao, R. Li, J. Palicot, H. Zhang, "Downlink interference minimization in cooperative cognitive LTE-femtocell networks", EURASIP Journal on Wireless Communications and Networking, 2013. [Online]. Available: https://doi.org/10.1186/1687-1499-2013194

[15] L. S. Yu, T. Ch. Cheng, Ch. K. Cheng, S. Ch. Wei, "Cognitive radio resource management for qos guarantees in autonomous femtocell networks", IEEE Int. Conf. Communications (ICC), 2010, pp. 1-6.

[16] I. Demirdogen, I. Guvenc, H. Arslan, "Capacity of closed-access femtocells networks with dynamic spectrum reuse", IEEE Int. Symposium on Personal Indoor and Mobile Communications (PIMRC), 2010, pp. 1315-1320. [Online]. Available: https://doi.org/10.1109/PIMRC.2010.5672015

[17] L. Huang, G. Zhu, X. Du, "Cognitive femtocell networks: an opportunistic spectrum access for future indoor wireless coverage", 
Wireless Commun., vol. 20, no. 2, pp. 44-51, 2013. [Online]. Available: https://doi.org/10.1109/MWC.2013.6507393

[18] Y. S. Soh, T. Q. S. Quek, M. Kountouris, G. Caire, "Cognitive hybrid division duplex for two-tier femtocell networks", IEEE Trans. Wireless Commun., vol. 12, no. 10, pp. 4852-4865, 2013. [Online]. Available: https://doi.org/10.1109/TWC.2013.090313.121264

[19] J. Lee, et al., "Traffic pattern-based opportunistic spectrum access of cognitive femto base stations for decentralized cross-tier interference management", Int. Conf. on Ubiquitous and Future Networks (ICUFN), 2014, pp. 352-356. [Online]. Available: https://doi.org/ 10.1109/icufn.2014.6876811

[20] F. Tariq, L. S. Dooley, A. S. Poulton, "An interference-aware virtual clustering paradigm for resource management in cognitive femtocell networks", Computers and Electrical Engineering, vol. 40, pp. 587598, 2014.

[21] P. Mach, Z. Becvar, "Distributed Hybrid spectrum sharing for OFDMA-based cognitive femtocells in 5G networks", in Proc. European Wireless, 2015, pp. 1-6.

[22] B. Ma, et al., Hybrid overlay/underlay cognitive femtocell networks: a game theoretic approach, IEEE Trans. Wireless Communications, vol. 14, no. 6, pp. 3259-3270, 2015. [Online]. Available: https://doi.org/10.1109/TWC.2015.2403363

[23] T. Yucek, H. Arslan, "A survey of spectrum sensing algorithms for cognitive radio applications", IEEE Communications Surveys \& Tutorials, vol. 11, no. 1, pp. 116-130, 2009. [Online]. Available: https://doi.org/10.1109/SURV.2009.090109

[24] J. Fan, Q. Yin, G. Y. Li, B. Peng, X. Zhu, "MCS selection for throughput improvement in downlink LTE systems", ICCCN, 2011. [Online]. Available: https://doi.org/10.1109/iccen.2011.6005743

[25] 3GPP TSG RAN WG4 (Radio) - R4-092042: Simulation assumptions and parameters for FDD HeNB RF requirements, 2009.

[26] Becvar, Z, et al., D21: Scenarios and requirements, Project TROPIC deliverable, 2013.

[27] Small Cell Forum, Interference Management in OFDMA Femtocells, 2011.

[28] T. Vanek, M. Rohlik, "Alternative protocols for femtocell backbone security", Wireless and Mobile Networking Conf., 2011, pp. 1-4. [Online]. Available: https://doi.org/10.1109/wmnc.2011.6097239 